\title{
A Rapid Method for Testing the Erythrocyte Sedimentation Rate
}

\author{
D M Dissanayake \\ Department of Pathology, Faculty of Medicine, University of Peradeniya
}

\begin{abstract}
The erythrocyte sedimentation rate (ESR) is one of the most common and traditional laboratory tests in the world for detecting acute and chronic inflammation. This simple test has served as a nonspecific indicator of presence of disease. It also has been found useful in monitoring response to therapy, especially in rheumatologic disease. It reflects both plasma concentration of acute-phase proteins of large molecular size and anaemia. The ESR test is easy to perform and inexpensive, is used today as a routine test worldwide. However, the ESR has some demerits, in requiring large volume of sodium citrate or EDTA blood and at least $1 \mathrm{hr}$ testing time. The minimum of $1 \mathrm{hr}$ testing time is not practical for modernized laboratories. In this paper we investigated the possibility of using a rapid ESR test done on an inclined tube. We compared the results obtained by the rapid method and the traditional Westergren method of ESR using Pearson correlation.

Blood samples from 153 patients with ESRs ranging from 1-165 were used for the study. Four $\mathrm{ml}$ of sodium citrate blood $(3.2 \mathrm{ml}$ of blood \& $0.8 \mathrm{ml}$ of $3.8 \%$ of sodium citrate) is prepared and two Westergren tubes filled. Both tubes are mounted on two separate Westergren tube racks. After mounting, one ESR tube is tilted to an angle of 45 degrees from vertical and readings were taken at $4,4.5,5,5.5,6,6.5,7,7.5,8,8.5,9,9.5,10,10.5,11.0,11.5,12,12.5$ and 13 minutes by noting the lowest point of the meniscus of clear plasma. The reading of the tube that is kept vertical is taken after 60 minutes. The statistical analysis showed that the values of the modified method between 10.5 to 11.5 minutes showed significant correlation with the values of the standard method. The correlation is significant for low ESR values as well as extremely high values.

The rapid ESR method has the advantage of being able to get a quick result. The accuracy of the results is acceptable. Same laboratory equipment can be used for the new method. This technique would be suitable for the rheumatology clinics where these test is requested frequently to modify the treatment.
\end{abstract}

Keywords: erythrocyte sedimentation rate, ESR.

Address for correspondence: D M Dissanayake, Department of Pathology, Faculty of Medicine, University of Peradeniya, Sri Lanka.

e-mail: dhammikamdisa@yahoo.co.uk 


\section{Introduction}

Inflammatory response to tissue injury (acute phase response) includes alteration in serum protein concentration, especially increase in fibrinogen, C-reactive protein and decrease in albumin. The changes occur in the acute inflammation and during active phase of chronic inflammation.

Measurement of the acute phase response is a helpful indicator of the presence and extent of inflammation and its response to treatment. Useful tests include estimation of C-reactive protein, fibrinogen and measurement of the erythrocyte sedimentation rate and plasma viscosity.

The erythrocyte sedimentation rate (ESR) is one of the most common and traditional laboratory tests in the world for detecting both acute and chronic inflammatory conditions and has served as a nonspecific indicator of presence of disease. It also has been found useful in monitoring response to therapy, especially in rheumatologic diseases and tuberculosis. Although ESR is a non specific test, there is a general impression that markedly elevated ESR may have a greater diagnostic specificity. Zacharski and Kyle reported from Mayo clinic that $58 \%$ of the patients with ESR of over $100 \mathrm{~mm} / \mathrm{hr}$ had underlying malignant disease. ${ }^{1}$ Many physicians have felt obliged to undertake extensive diagnostic evaluation of patients found to have extremely high ESR. Extreme elevations were found in several conditions including malignancies, infections and collagen vascular diseases. It reflects both plasma concentration of acute-phase proteins of large molecular size and anaemia. ${ }^{2}$

This method is easy to perform and inexpensive. It has become popular and it's procedures are internationally standardized. ${ }^{2}$ Therefore it is used today as a routine test worldwide. However, the ESR has some demerits, in requiring a large volume of sodium citrate or EDTA blood and at least $1 \mathrm{hr}$ testing time. When ESR is tested, incorrect results are obtained if the tube is not stood straight-up and vertically. ${ }^{3}$ Both reading error of meniscus line and temperature at the testing site cause inaccuracy. ${ }^{4}$ The 1 hour testing time is not practical for modernized laboratories.

In this paper we investigated the possibility of using a rapid ESR done in an inclined tube. We compared the results obtained by the rapid method and traditional Westergren method.

\section{Materials and methods}

Residual blood from 156 patient samples which were referred to the haematology laboratory of teaching hospital Peradeniya for routine haematological investigations was utilized for the study only after completing all the requested investigations and dispatching the respective reports.

As a routine investigation, assessment of ESR had been already performed on all the samples, using standard Westergren method. Accordingly, ESR values in the study sample ranged from 1 to 165 (Graph 1). The assessment of ESR was then performed following the modified new method described below. Parallel with the experimental tube, a second ESR testing (control) was performed for each sample using standard Westergren method for the purposes of 
comparison. The experimental tube was tilted at an angle of 45 degrees just after mounting, while the second tube or the control remained strait. Readings were recorded at every 30 seconds from 4 minutes to 13 minutes in the tilted tube by reading the lowest level of the meniscus. The reading of the tube that was kept vertical was taken after 60 minutes. The relationship of the two readings was assessed by Pearson correlation using the Minitab statistical package.

Graph 1. Distribution of ESR values in the sample used in the study.

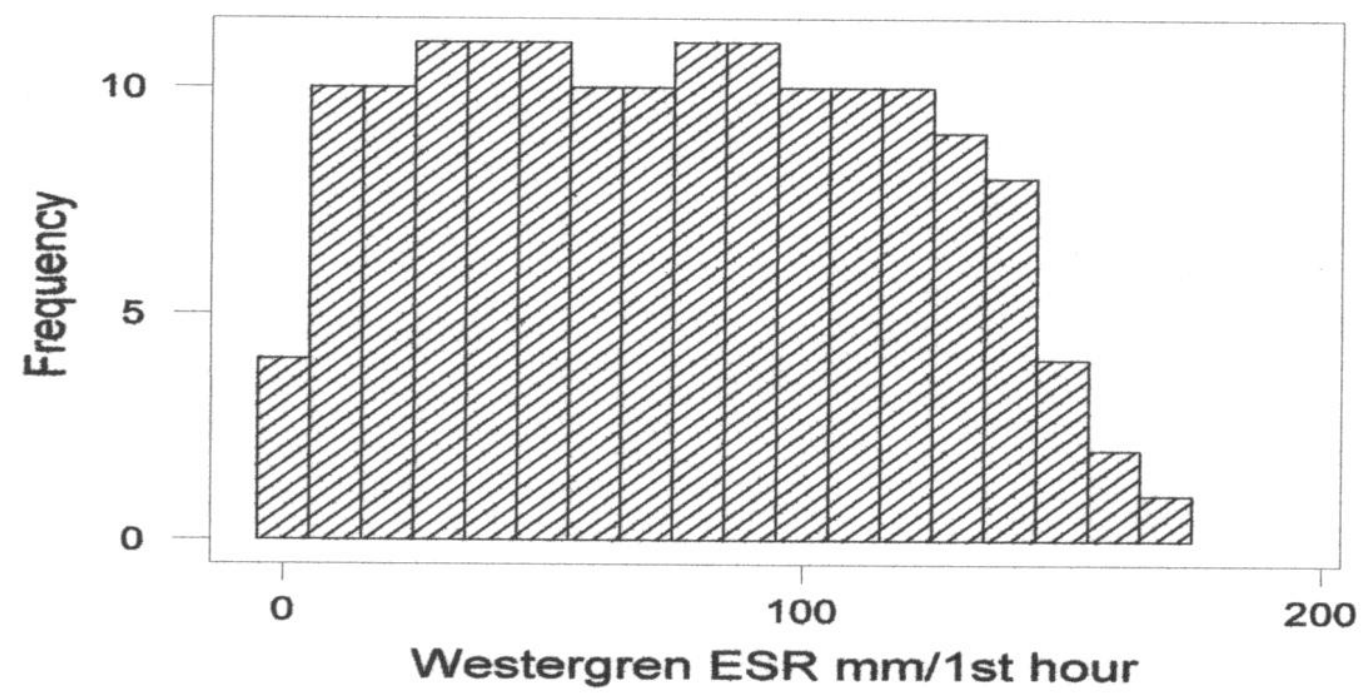

\section{Results}

The correlation of ESR from the standard Westergren method gradually increased with time till 10 minutes and reached a plateau between 10-11.5 minutes (Graph 2). The maximum correlation ( $r$ ) was 0.986 at 10.5 minutes (Graph 3), 0.985 at 11 minutes and 0.986 at 11.5 minutes. The $r$ value gradually declined after 11.5 minutes. The correlation is significant for low ESR values as well as for extremely high values. 
Graph 2: Correlation (r) with the time in minutes.

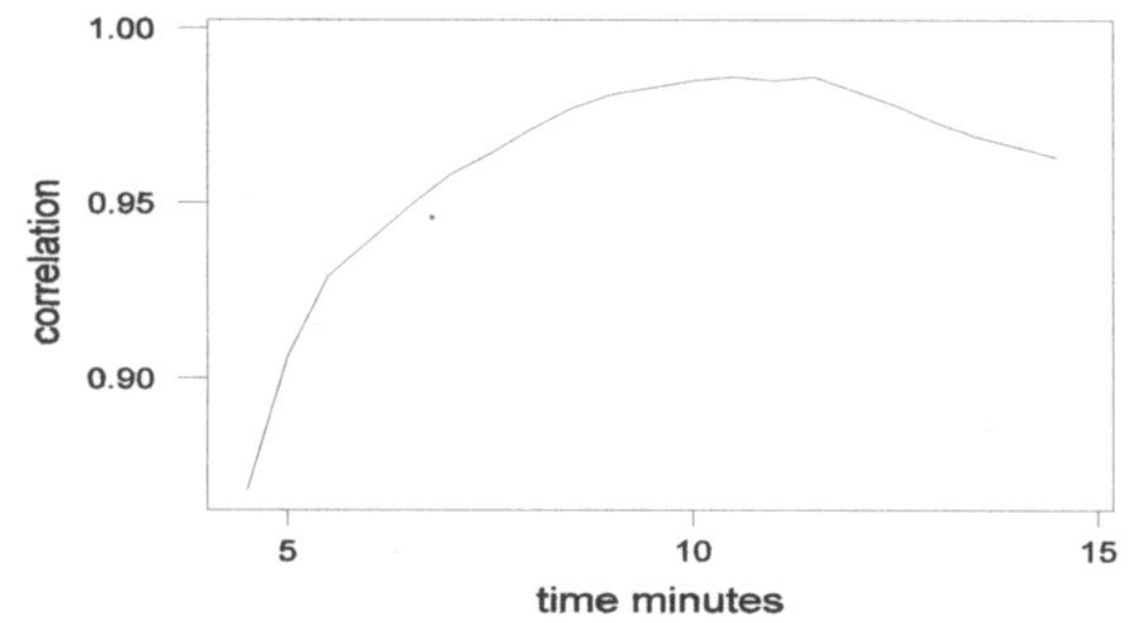

Graph 3. Regression curve of results of rapid method (at 10.5 minutes) and Westergren method $(r=0.986)$

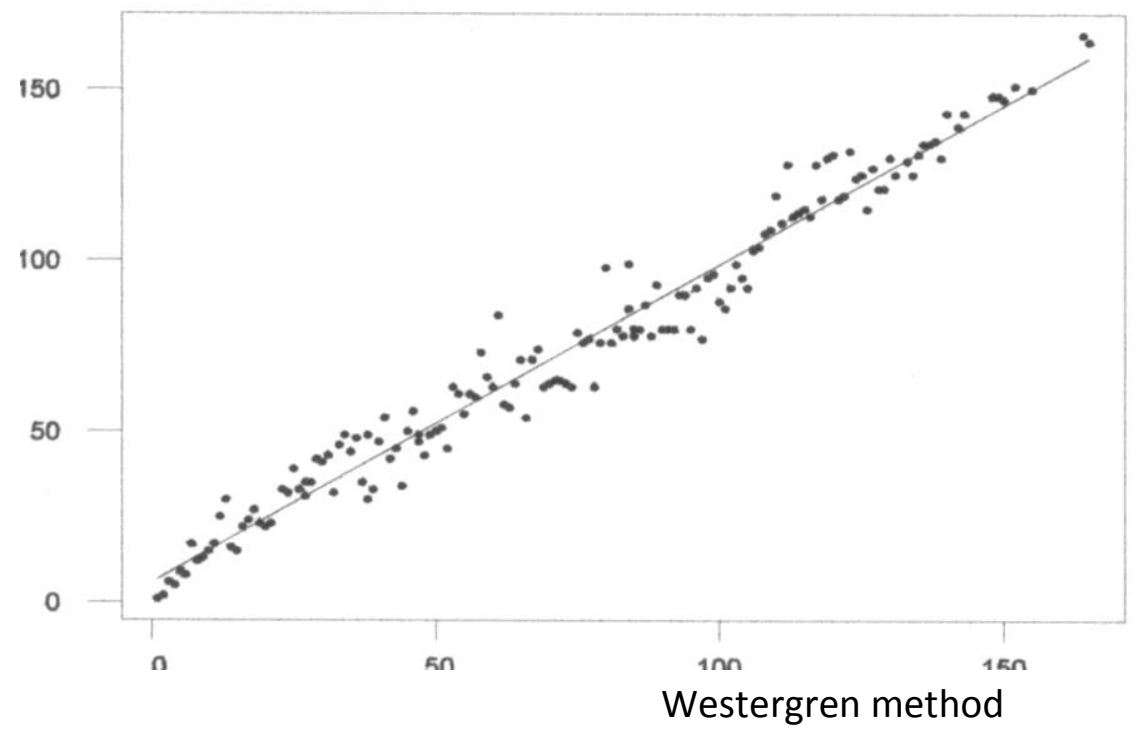

\section{Conclusion}

Erythrocyte sedimentation can be observed to take place in three stages: a preliminary stage of at least a few minutes during which time rouleaux formation occur and aggregates form; then a period in which the sinking of the aggregates take place at approximately a constant speed, ${ }^{5,6}$ and finally a phase during which the rate of sedimentation slows as the aggregated cells pack at the bottom of the tube. It is obvious that the longer the tube used the longer the second period can last and greater the sedimentation rate appear to be. This is an advantage of the 
Westergren tube. With a shorter tube, e.g. a Wintrobe tube, packing may start before an hour has elapsed.

As rouleaux formation and aggregation step is essential we can shorten the time by reducing the time necessary for the sinking of aggregates and for packing. By inclining the tube to 45 degrees we can significantly shorten the time required for the sinking of the aggregates by shortening the distance for the sinking.

Excessively short testing time cannot yield sufficient aggregation of red cells and devices with short testing time theoretically cannot provide accurate data because of insufficient aggregation. $^{7}$

This method still carries disadvantages like, having same biohazard risk as the original Westergren method, and needs $2 \mathrm{ml}$ of blood for the procedure. The correlation is significant for low ESR values as well as for extremely high values. No special laboratory equipment is necessary. This technique would be suitable for the rheumatology clinics and when and where this test is requested frequently to modify the treatment.

\section{Acknowledgement}

Technical assistance by Mrs C.K. Rajanayake is acknowledged.

\section{References}

1. Zacharski LR, Kyle RA. Significance of extreme elevation of erythrocyte sedimentation rate. JAMA. 1967; 202: 264-6.

2. International Council for Standardization in Haematology (Expert panel on blood Rheology). ICSH recommendations for measurement of erythrocyte sedimentation rate. J Clin Pathol. 1993; 46: 198-203.

3. Dobashi $\mathrm{T}$, Idonuma A, Toyama $\mathrm{Y}$ et al. Effect of concentration on enhanced sedimentation rate of erythrocytes in an inclined vessel. Biorheology. 1994; 31: 383-93.

4. Manley RW. The effect of room temperature on erythrocyte sedimentation rate and its correction. J Clin Pathol. 1957; 10: 354-6.

5. Fabry TL. Mechanism of erythrocyte aggregation and sedimentation. Blood. 1987; 70: 1572-6.

6. Reinhart WH, Singh A, Straub PW. Red blood cell aggregation and sedimentation rate: The role of red cell shape. Br J Haematol. 1989; 73: 551-6.

7. Tabuchi T, Tominaga H, Tatsumi N. Problems related to rapid methods for erythrocyte sedementation rate test and their solution. Southeast Asian J Trop Med Publ Health. 2002; 33: 151-4. 